\title{
Construction of 1D Heterostructure NiCo@ C/ZnO Nanorod with Enhanced Microwave Absorption
}

Cite as

Nano-Micro Lett.

(2021) $13: 175$

Received: 3 June 2021

Jianwei Wang ${ }^{1}$, Zirui Jia ${ }^{1,2,3}$, Xuehua Liu ${ }^{1}$, Jinlei Dou ${ }^{1}$, Binghui Xu ${ }^{1}$, Bingbing Wang ${ }^{1}$, Guanglei $\mathrm{Wu}^{1} \bowtie$

Accepted: 25 July 2021

Published online: 16 August 2021

(C) The Author(s) 2021

\section{HIGHLIGHTS}

- NiCo-LDHs successfully grow in situ on rod-like $\mathrm{ZnO}$ with unique structures.

- The interface of NiCo@ $\mathrm{C}$ and rod-like $\mathrm{ZnO}$ result in interfacial polarization.

- The RLmin of NiCo@C/ZnO reaches - $60.97 \mathrm{~dB}$ with the EABmax of $6.08 \mathrm{GHz}$.

- The excellent performance comes from the effect of dielectric loss and magnetic loss.

\begin{abstract}
Layered double hydroxides (LDHs) have a special structure and atom composition, which are expected to be an excellent electromagnetic wave (EMW) absorber. However, it is still a problem that obtaining excellent EMWabsorbing materials from LDHs. Herein, we designed heterostructure NiCo-LDHs@ZnO nanorod and then subsequent heat treating to derive $\mathrm{NiCo@C/ZnO} \mathrm{composites.}$ Finally, with the synergy of excellent dielectric loss and magnetic loss, an outstanding absorption performance could be achieved with the reflection loss of $-60.97 \mathrm{~dB}$ at the matching thickness of $2.3 \mathrm{~mm}$, and the widest absorption bandwidth of $6.08 \mathrm{GHz}$ was realized at $2.0 \mathrm{~mm}$. Moreover, this research work provides a reference for the development and utilization of LDHs materials in the field of microwave absorption materials and can also provide ideas for the design of layered structural absorbers.
\end{abstract}
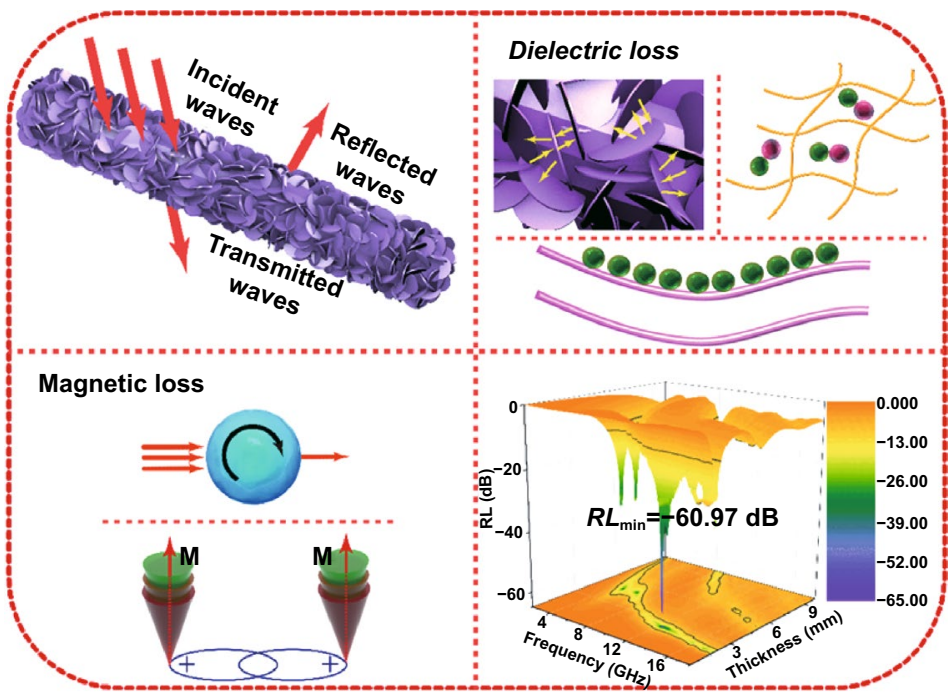

KEYWORDS Layered double hydroxides; NiCo@C/ZnO; Interface polarization; Microwave absorption

Jianwei Wang and Zirui Jia have contributed equally to this work.

$\bowtie$ Guanglei Wu, wuguanglei@qdu.edu.cn; wuguanglei@mail.xjtu.edu.cn

1 State Key Laboratory of Bio-Fibers and Eco-Textiles, Institute of Materials for Energy and Environment, College of Materials Science and Engineering, Qingdao University, Qingdao 266071, People's Republic of China

2 College of Chemistry and Chemical Engineering, Qingdao University, Qingdao 266071, Shandong, People's Republic of China

3 Weihai Innovation Institute, Qingdao University, Weihai 264200, Shandong, People's Republic of China 


\section{Introduction}

Nowadays, electromagnetic waves (EMW) are currently used in a wide range of medical and military equipment as a medium for the wireless control and transmission of information in electronic devices [1-3]. However, the failure of electronic facilities, human organ damage and other negative effects caused by electromagnetic pollution deserve our attention $[4,5]$. In order to make better prevention of EMW, EMW-absorbing materials that can attenuate EMW energy and convert incident EMW into heat or otherwise forms of energy have been designed and developed [6, 7]. The idealized absorbing material should meet the requirement of wide bandwidth, light weight and thin thickness [8-10]. However, the EMW absorption capacity of a single material is relatively weak. Therefore, the development of composites that gratify the demands will become the focus of future studies.

Many suitable absorbers have been developed, such as carbon materials [11], alloys [12], transition metal oxides/ sulfides/selenides [13-15], and MXene [16]. With the development of technology, the requirements for the EMW absorption properties and the condition of using EMWabsorbing materials are become more and more stringent. So, it is necessary to develop better materials to meet the requirements of practical application. At this point, metal alloys came into the research range due to excellent permittivity and permeability, with NiCo alloys being the most notable $[17,18]$. NiCo alloys possess the characteristics of high-temperature calcination resistance, oxidation resistance, corrosion resistance, and easy extraction [19, 20], and are widely used in high-tech fields such as aerospace.

Metal organic frameworks (MOFs) are a new type of material formed by the self-assembly of metal cations with organic ligands, which have the advantages of large surface area, low density, and high porosity [21-24]. However, individual MOFs have not yet been extensively applied in EMW-absorbing materials as a result of poor dielectric properties [25]. Therefore, many attempts have been made to tune the electromagnetic properties of MOF materials and derivatives to obtain satisfactory EMW absorption performance. The most popular approach is introducing other metal cations to modulate the morphology and elemental composition of MOFs and derivatives [26, 27]. Che et al. [28] prepared $\mathrm{Ni} / \mathrm{C} / \mathrm{ZnO}$ microspheres with yolk-shell structure based on bimetallic NiZn-MOFs. Zhou et al. [29] prepared CoZn-MOFs as templates to prepare rod-shaped $\mathrm{Co} / \mathrm{ZnO} / \mathrm{C}$ composites, all of which can obtain excellent EMW-absorbing properties. However, there are still some difficulties, such as complicated preparation process and poor attenuation performance, etc. Therefore, it is still necessary to find other ideas to obtain ideal EMW-absorbing materials [30-32].

Layered double hydroxides (LDHs) are compounds consisting of positively charged lamellae and interlayer anions interacting with each other [33-35]. Generally, the most common methods for generating LDHs include hydrothermal method, ion exchange method, co-precipitation method, and so on [36, 37]. Metallic alloys are acquired via hightemperature calcination of LDHs materials in an inert gas environment, which is one of the methods to attain polymetallic alloys. The method is an important way to access excellent electromagnetic absorbing materials and make the electromagnetic parameters of the alloys controllable [38, 39]. Zinc oxide $(\mathrm{ZnO})$ is a semiconductor material with a wide band gap and excellent dielectric properties, which, together with its ease of making unique structures, makes $\mathrm{ZnO}$ promising for development as dielectric material [40, 41].

In this paper, we presented a novel design that exploits the synergistic effect between components, which to develop electromagnetic absorbing materials that can meet practical needs. Hence, NiCo@C/ZnO composites decorated with NiCo alloy particles were synthesized through hydrothermal method and annealing process, which demonstrated excellent EMW absorption performance. The minimum reflection loss $\left(R L_{\text {min }}\right)$ value was $-60.97 \mathrm{~dB}$ with thickness of $2.3 \mathrm{~mm}$, and the maximum effective absorption bandwidth $\left(\mathrm{EAB}_{\max }\right)$ value was $6.08 \mathrm{GHz}$ with thickness of $2.0 \mathrm{~mm}$. In addition, the electromagnetic absorption mechanism of $\mathrm{NiCo@C/ZnO}$ composites was discussed in detail. This work not only provides ideas for the development and utilization of MOFs materials, but also serves references for the design of new electromagnetic absorbing materials.

\section{Experimental Section}

\subsection{Chemical Reagents}

All chemicals were of analytical grade (AR) and used directly without any further purification. Cobalt (II) nitrate 
hexahydrate $\left(\mathrm{Co}\left(\mathrm{NO}_{3}\right)_{2} \cdot 6 \mathrm{H}_{2} \mathrm{O}, \mathrm{AR}, 99 \%\right)$, nickel (II) nitrate hexahydrate $\left(\mathrm{Ni}\left(\mathrm{NO}_{3}\right)_{2} \cdot 6 \mathrm{H}_{2} \mathrm{O} \mathrm{AR}, 99 \%\right)$, ammonia solution $\left(\mathrm{NH}_{3} \cdot \mathrm{H}_{2} \mathrm{O}, 28 \%\right)$, Zinc acetate $\left(\mathrm{Zn}\left(\mathrm{CH}_{3} \mathrm{COO}\right)_{2}, \mathrm{AR}\right.$, 99-101\%), 2-methlimidazole $\left(\mathrm{C}_{4} \mathrm{H}_{6} \mathrm{~N}_{2}, 98 \%\right.$, 2-MIM), hexamethylene tetramine $\left(\mathrm{C}_{6} \mathrm{H}_{12} \mathrm{~N}_{4}\right)$, absolute ethanol $\left(\mathrm{C}_{2} \mathrm{H}_{6} \mathrm{OH}\right)$, and methyl alcohol $\left(\mathrm{CH}_{3} \mathrm{OH}, 99.5 \%\right)$. They are purchased from Chemical Reagents Company Limited of China Pharmaceutical Group. Deionized water was used throughout the experiment.

\subsection{Sample Preparation}

\subsubsection{Preparation of Rod-Like $\mathrm{ZnO}$}

As shown in Scheme 1, zinc acetate and hexamethylenetetramine were added to $50 \mathrm{~mL}$ of deionized water at the ratio of 1:1 and stirred with a glass rod until the particles were completely dissolved. The $\mathrm{pH}$ of the mixed solution was adjusted to $\mathrm{pH}=10$ with ammonia and stirred continuously at room temperature for about $3 \mathrm{~h}$. The solution was transferred to $100 \mathrm{~mL}$ of PTFE liner and kept at $90{ }^{\circ} \mathrm{C}$ for $12 \mathrm{~h}$. Finally, after cooling to room temperature, the sample was dried at $60{ }^{\circ} \mathrm{C}$ after washing with deionized water and ethanol. The rod-like $\mathrm{ZnO}$ materials were defined as $\mathrm{S}-1$.

\subsubsection{Preparation of NiCo@C Composites}

$0.5 \mathrm{~g}$ of $\mathrm{Co}\left(\mathrm{NO}_{3}\right)_{2} \cdot 6 \mathrm{H}_{2} \mathrm{O}$ and $0.5 \mathrm{~g}$ of $\mathrm{Ni}\left(\mathrm{NO}_{3}\right)_{3} \cdot 6 \mathrm{H}_{2} \mathrm{O}$ were added to $40 \mathrm{~mL}$ of methanol solution which was recorded as solution A. $8 \mathrm{mmol}$ of 2-methylimidazole (2-MIM) was dissolved in $20 \mathrm{~mL}$ of methanol solution and stirred until the pellet was completely dissolved, which was recorded as solution B. Then, solution B was quickly poured into solution A,

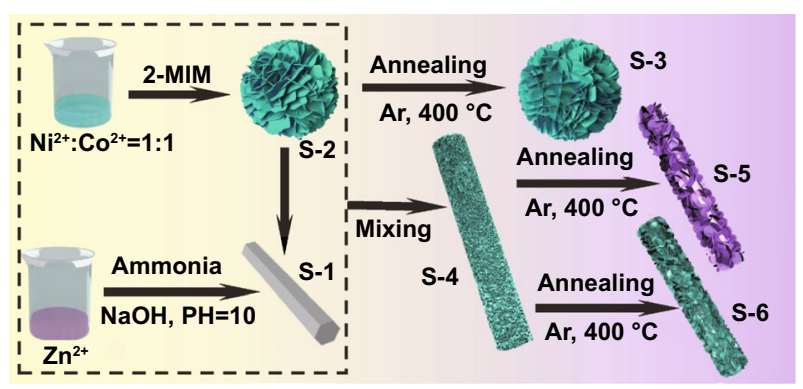

Scheme 1 Illustration of the synthetic process of composites and stirred continuously at room temperature for $2 \mathrm{~h}$. Then, the solution was transferred to a $100 \mathrm{~mL}$ PTFE liner and maintained at $160{ }^{\circ} \mathrm{C}$ for $12 \mathrm{~h}$. After cooling to room temperature, the resulting material is washed several times with deionized water and ethanol and then dried at $60{ }^{\circ} \mathrm{C}$. The samples were denoted as S-2. The material was calcined at $400{ }^{\circ} \mathrm{C}$ for $3 \mathrm{~h}$ under argon atmosphere at a heating rate of 5 ${ }^{\circ} \mathrm{C} \min ^{-1}$. The obtained composites were designated as S-3.

\subsubsection{Preparation of Rod-Like Structured NiCo@C/ZnO Composites}

Typically, $1.0 \mathrm{~g}$ of rod-like $\mathrm{ZnO}$ material is added to $40 \mathrm{~mL}$ methanol and stirred to obtain a homogeneous solution. $0.5 \mathrm{~g}$ of $\mathrm{Co}\left(\mathrm{NO}_{3}\right)_{2} \cdot 6 \mathrm{H}_{2} \mathrm{O}$ and $0.5 \mathrm{~g}$ of $\mathrm{Ni}\left(\mathrm{NO}_{3}\right)_{3} \cdot 6 \mathrm{H}_{2} \mathrm{O}$ were joined in the mixed solution. Then $8 \mathrm{mmol}$ of 2-Methylimidazole (2-MIM) was dissolved in $20 \mathrm{~mL}$ of methanol solution with stirring until the pellet was fully dissolved. The 2-methylimidazole solution was quickly poured into a methanolic solution containing rod-like $\mathrm{ZnO}$ materials and stirred continuously for $2 \mathrm{~h}$ at room temperature. Then, the solution was transferred to a $100 \mathrm{~mL}$ PTFE liner and maintained at $160{ }^{\circ} \mathrm{C}$ for $12 \mathrm{~h}$. After cooling to room temperature, the resulting material is washed several times with deionized water and ethanol and then dried at $60{ }^{\circ} \mathrm{C}$. The samples were denoted as S-4. Finally, the composites were calcinated at $400{ }^{\circ} \mathrm{C}$ under Ar atmosphere and Air atmosphere with a heating rate of $5{ }^{\circ} \mathrm{C} \mathrm{min}^{-1}$ for $3 \mathrm{~h}$. The as-obtained composites were named as S-5 and S-6, respectively.

\subsection{Characterization}

The crystalline structures of samples were obtained on powder X-ray diffraction (XRD, Rigaku Ultima IV with $\mathrm{Cu}$-Ka radiation $(\lambda=0.15418)$ ). The morphology of samples was characterized by a field emission scanning electron microscope (SEM, JFOL JSM-7800F) equipped with an energy-dispersive spectrum. The crystal structure and microstructure of samples were recorded by a transmission electron microscope (TEM, JEOL JEM-2100). Raman shift of samples was collected through using a Renishaw in Via Plus Micro-Raman spectroscopy system with a 50-mW laser at $532 \mathrm{~mm}$. The bond states of surface elements for samples were tested by X-ray photoelectron spectroscopy (XPS) on Thermo Fisher ESCALAB 250Xi spectrometer with an 
Al Ka X-ray source $(1486.6 \mathrm{eV})$. The thermogravimetric analysis (TGA) of samples was recorded by an SDT Q600 analyzer from room temperature to $900{ }^{\circ} \mathrm{C}$ with a heating rate of $10{ }^{\circ} \mathrm{C} \mathrm{min}{ }^{-1}$ under $\mathrm{Ar}$ atmosphere.

\subsection{Electromagnetic Parameters}

Based on the mass ratio of $1: 2$, the composites and paraffin are mixed evenly, and pressed into a ring sample to be tested, in which the inner diameter, outer diameter, and thickness are 3.04, 7.00, and $2.00 \mathrm{~mm}$, respectively. The EM parameters were obtained by a vector network analyzer (N5234A, Agilent, USA) using a coaxial method in the frequency range of $2.0-18.0 \mathrm{GHz}$. Using transmission line theory is to work out the reflection loss $(R L)$ values of absorbers at different thicknesses in their corresponding frequency $[42,43]$.

$Z_{\text {in }}=Z_{0} \sqrt{\mu_{r} / \varepsilon_{r}} \tanh \left|j\left(\frac{2 \pi f d}{c}\right) \sqrt{\varepsilon_{r} \mu_{r}}\right|$

$R L(\mathrm{~dB})=20 \log \left|\frac{Z_{\text {in }}-Z_{0}}{Z_{\text {in }}+Z_{0}}\right|$

where $c, f$ and $d$ refer to the light speed, the corresponding frequency and the matching thickness, $Z_{\text {in }}$ is the normalized input impedance and the $Z_{0}$ means the impedance of free space, $\varepsilon_{r}$ and $\mu_{r}$ are the complex permittivity and complex permeability, respectively. Normally, when $R L<-10 \mathrm{~dB}$, it means that the absorbing material will absorb more than $90 \%$ of the incident EMW.

\section{Results and Discussion}

\subsection{Composition and Structure}

The crystal structures of all samples were analyzed by XRD. As displayed in Fig. 1, the curve of S-1 shows the XRD pattern of rod-like $\mathrm{ZnO}$ with characteristic peaks of $31.8^{\circ}$, $34.4^{\circ}, 36.3^{\circ}, 47.5^{\circ}, 56.6^{\circ}$, and $62.9^{\circ}, 66.4^{\circ}$, assigning to the (100), (002), (101), (102), (110), and (103), (200) of $\mathrm{ZnO}$ crystal planes. There are obvious characteristic peaks on the curve of $\mathrm{S}-2$, where the characteristic peaks at $11.3^{\circ}$, $22.6^{\circ}, 33.9^{\circ}, 38.1^{\circ}$ and $60.1^{\circ}$ conform to the (003), (006), (100), (015), and (110) crystal planes of NiCo-LDHs, respectively [44]. The characteristic peaks corresponding to the (111), (200), and (220) crystals of $\mathrm{Ni}$ are $44.6^{\circ}$, $51.8^{\circ}$ and $75.9^{\circ}$ on the curves belonging to S-3 (PDF No. 4-0850). However, compared with the standard cards Co (PDF No. 15-0806) and Ni (PDF No. 4-0850), the position of the characteristic peak is somewhat shifted, which proves that the nickel-cobalt alloy is formed instead of a single metal. In Fig. S1, in addition to the characteristic peaks of NiCo alloys, characteristic peaks attributed to amorphous $\mathrm{C}$ can also be seen $[11,12]$, proving that NiCo@C can be obtained from NiCo-LDHs composites through high-temperature calcination in Ar. In the XRD spectrum of S-4, the characteristic peaks of NiCo-LDHs can be seen in addition to those of $\mathrm{ZnO}$, which suggest the successful preparation of NiCo-LDHs@ZnO composites. NiCo@C/ZnO composites obtained via calcination of NiCo-LDHs@ZnO composites

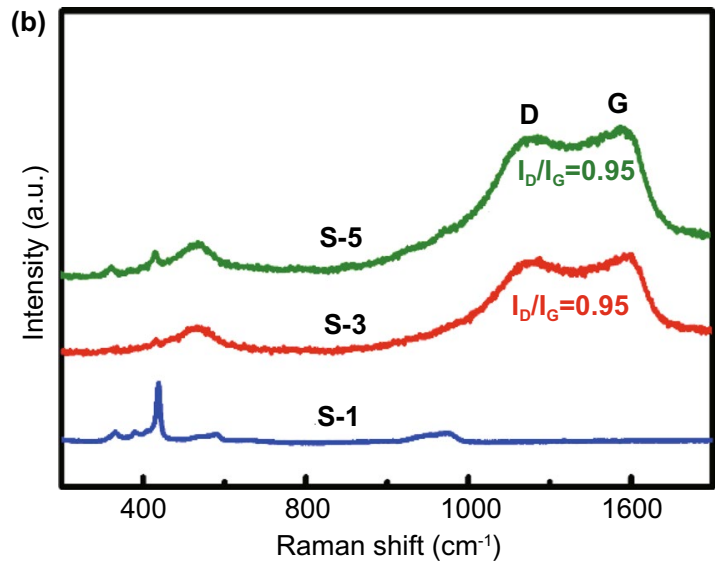

Fig. 1 a XRD patterns of all samples, b Raman spectrum of S-1, S-4, and S-5

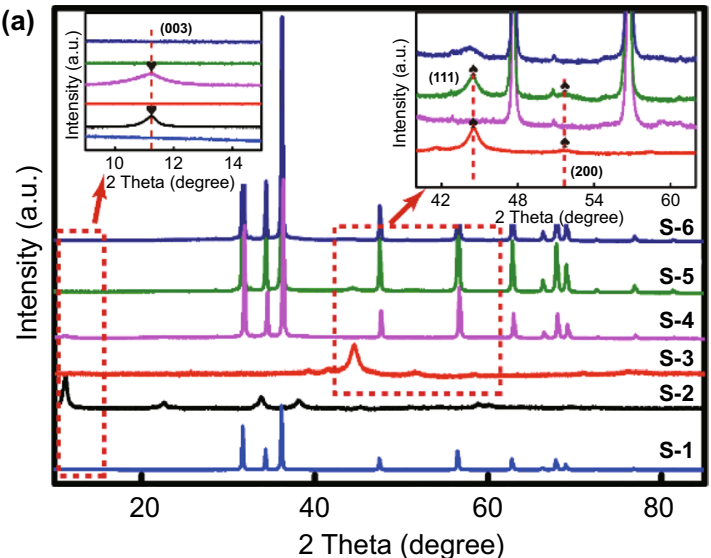


under Ar atmosphere are evidenced by the distinct peaks of $\mathrm{ZnO}$ and NiCo alloys in the XRD spectrum of S-5. The XRD spectrum of S-6 provides characteristic peaks of $\mathrm{ZnO}$ and $\mathrm{Co}_{2} \mathrm{NiO}_{4}$ from $\mathrm{Co}_{2} \mathrm{NiO}_{4} @ \mathrm{ZnO}$ composites. No significant characteristic peaks of impurities were found in all the XRD patterns, confirming the high purity of the prepared samples. Figure $1 b$ shows the Raman spectra of S-1, S-3, and S-5. The characteristic peak of $\mathrm{ZnO}\left(437 \mathrm{~cm}^{-1}\right)$ appears on the curve of S-1. The characteristic peak of NiCo $\left(534 \mathrm{~cm}^{-1}\right)$ and obvious $\mathrm{D} / \mathrm{G}$ peaks were noted in $\mathrm{S}-3$, where the $I_{\mathrm{D}} / I_{\mathrm{G}}$ value was 0.95 , which proves that graphitic carbon is produced during calcination at high temperatures. $\mathrm{NiCo} / \mathrm{C}$ composites are favorable for the formation of conductive networks and the construction of heterogeneous interfaces. S-5 has both characteristic peaks of NiCo alloy, $\mathrm{ZnO}$ and obvious $\mathrm{D} / \mathrm{G}$ peaks, in which the $I_{\mathrm{D}} / I_{\mathrm{G}}$ value does not change, and this conclusion is also consistent with the XRD results.

The SEM images reveal the external morphology and elemental distribution of the sample. Figure 2 a gives an
SEM image of $\mathrm{ZnO}$, which has a relatively homogeneous morphology and is not connected to each other. The cross section of the rod-shaped $\mathrm{ZnO}$ is ortho-hexagonal as seen in Fig. 2a-1. SEM image of NiCo-LDHs (S-2) (Fig. 1b) indicates the flower-like structures of NiCo-LDHs generated by stacking multiple lamellar structures, in which the size of each nanosheet is greater than $1 \mu \mathrm{m}$. It can be seen from the elemental distribution map of S-2 that the elements $\mathrm{O}, \mathrm{Ni}$ and $\mathrm{Co}$ are uniformly dispersed in the NiCo-LDHs. The comparison of SEM images of S-2 (Fig. 2b) with S-3 (Fig. 2c) demonstrates no significant change in the overall morphology after high temperature annealing under $\mathrm{Ar}$ atmosphere, it underwent a transition from NiCo-LDH to NiCo@C, which led to the thinning of the nanosheets in S-3, the EDS spectrum (Fig. S2) indicate that this material is composed of three elements: nickel, cobalt and carbon, and their mass percentages are $38.42 \%, 33.96 \%$, and $27.63 \%$, respectively. In the SEM image of NiCo-LDHs@ $\mathrm{ZnO}$ (S-4) (Fig. 2d), the surface of rod-like $\mathrm{ZnO}$ is covered
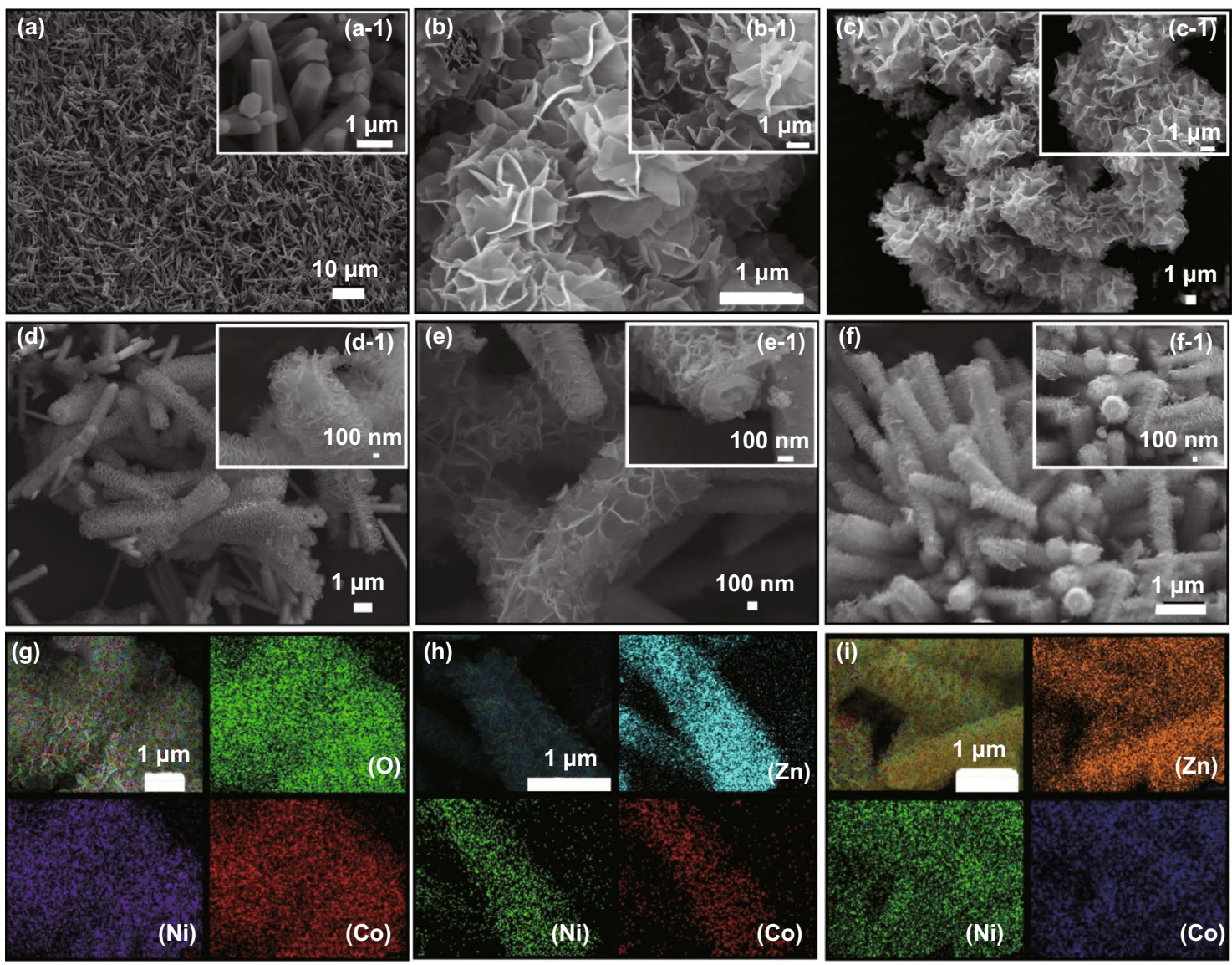

Fig. 2 SEM images of (a, a-1) S-1, (b, b-1) S-2, (c, c-1) S-3, (d, d-1) S-4, (e, e-1) S-5, (f, f-1) S-6 and elemental mapping distribution of g S-2, h S-4, i S-5 
with many layers of NiCo-LDHs and the layers are closely aligned. $\mathrm{Zn}$ elements are relatively concentrated, while $\mathrm{Ni}$ and Co elements are mainly scattered around the rod-like $\mathrm{ZnO}$ in the elemental distribution diagram. In Fig. 2e, the NiCo@C/ZnO composite (S-5) obtained after calcination at high temperature has a significantly lower number of layers coated on the rod-like $\mathrm{ZnO}$. In the elemental distribution diagram (Figs. $2 \mathrm{i}$ and S3b), the distribution of $\mathrm{Zn}$ elements does not change obviously, while the distribution of $\mathrm{Ni}$ and Co elements becomes more dispersed and found that the contents of $\mathrm{C}, \mathrm{O}, \mathrm{Ni}, \mathrm{Co}$, and $\mathrm{Zn}$ were $15.03 \%, 14.43 \%$, $14.76 \%, 11.92 \%$, and $43.85 \%$ (Fig. S3b), respectively. The size of the layered structure of S-6 decreases significantly, but the number of layers remains almost unchanged (Fig. 2f).

The TEM images allow further to observe and analyze the internal structure and material composition of the prepared samples (Fig. 3). The rod-like $\mathrm{ZnO}$ exhibits a diameter of approximately $500 \mathrm{~nm}$ with no connections among themselves (Fig. 3a), which is consistent with observation in the SEM images (Fig. 2a). The lattice spacing $(d)$ observed in the HR-TEM image of $\mathrm{ZnO}$ is $0.266 \mathrm{~nm}$, matching the (002) crystal plane of $\mathrm{ZnO}$ (Fig. 3a-1). In Fig. 3a-2, the spots are uniformly dispersed and distributed in order, demonstrating the single-crystal hexagonal structure of $\mathrm{ZnO}$. The layered structure overlying the rod structure can still be visualized in Fig. 3b, with the shaded part of the figure shows the NiCoLDHs overlying the rod-like $\mathrm{ZnO}$. The lattice with a spacing of $0.262 \mathrm{~nm}$ in Fig. 3b-1 corresponds to the (012) crystal plane of NiCo-LDHs [45]. The observed circle points to the (107) crystal plane of NiCo-LDHs in high-resolution TEM images of S-4 (Fig. 3b-2,). The lamellar structure changes in size and number after high-temperature annealing (Fig. 3c), and a number of grains (NiCo alloy) can also be observed. Besides, the lattice spacing of $0.204 \mathrm{~nm}$ (Fig. 3c-1) matches (111) plane of the NiCo alloy, which also confirms the formation of NiCo alloy [46, 47]. The diffraction rings in Fig. 3c-2 are attributed to the (111), (200), (220), and (311) crystal planes of the NiCo alloy, which are in accordance with the results of other characterizations.

In order to further analyze the weight variation trend of the samples during calcination, TGA measurements were made in $\mathrm{Ar}$ atmosphere at a temperature increasing rate of $10{ }^{\circ} \mathrm{C} \mathrm{min}{ }^{-1}$ from room temperature to $900{ }^{\circ} \mathrm{C}$. In Fig. S4, the mass of S-4 decreased slightly before $200{ }^{\circ} \mathrm{C}$ by about $0.99 \%$, which is due to the gradual evaporation of the water contained in the sample with the increase in temperature. From 200 to $720^{\circ} \mathrm{C}$, the mass of the sample decreases linearly with the increase in temperature, and the final mass
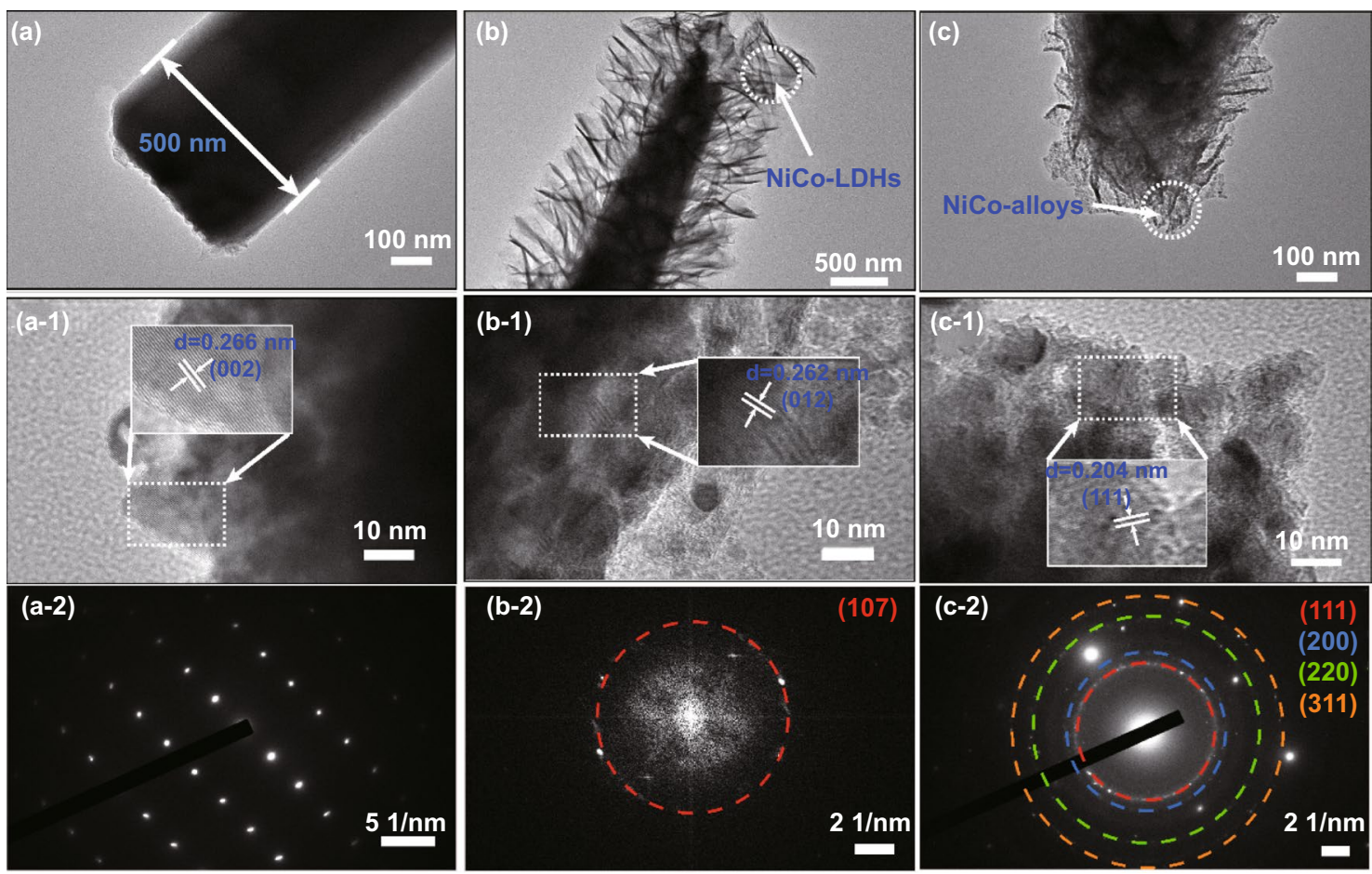

Fig. 3 TEM images and SAED patterns of (a, a-1, a-2) S-1, (b, b-1, b-2) S-4, (c, c-1, c-2) S-5 
decreases to $13.05 \%$, which was due to the gradual decomposition of NiCo-LDHs into NiCo@C composites with the increase in temperature. From the full-scan XPS spectrum (Fig. 4a), there are five characteristic peaks in S-5, which belong to C $1 \mathrm{~s}, \mathrm{Zn} 2 \mathrm{p}, \mathrm{O} 1 \mathrm{~s}$, Co 2p, and Ni 2p, respectively. In the high-resolution spectra in $\mathrm{C} 1 \mathrm{~s}$ (Fig. 4b), the characteristic peaks located at 284.6, 286.5, and $288.5 \mathrm{eV}$ ascribed to $\mathrm{C}-\mathrm{C} / \mathrm{C}=\mathrm{C}, \mathrm{C}-\mathrm{O}$, and $\mathrm{C}-\mathrm{C}=\mathrm{O}$, respectively [48]. From the high-resolution spectra of $\mathrm{Zn} 2 \mathrm{p}$ (Fig. 4c), the characteristic peak at $1021.4 \mathrm{eV}$ corresponds to $\mathrm{Zn} 2 \mathrm{p}_{3 / 2}$ and the characteristic peak at $1044.6 \mathrm{eV}$ corresponds to $\mathrm{Zn} 2 \mathrm{p}_{1 / 2}$. On the high-resolution spectrum of O 1 s of S-5 (Fig. 4d), three characteristic peaks of 531.5, 530.6, and $529.0 \mathrm{eV}$ can be observed, corresponding to the water or $\mathrm{O}$ adsorbed on the sample surface, oxygen vacancies and metal-O (Ni-O/ Co-O) respectively $[49,50]$. Co $2 p$ can be fitted to six distinct characteristic peaks (Fig. 4e), the characteristic peaks at 779.2 and $795.4 \mathrm{eV}$ ascribed to metal-Co, and the characteristic peak at 780.6 and $796.3 \mathrm{eV}$ can match the Co-O bond. The remaining characteristic peaks at 788.3 and $803.4 \mathrm{eV}$ can be matched to the satellite peaks $[51,52]$. In the Ni $2 p$ high-resolution XPS spectrum of S-5 (Fig. 4f), the peaks at 878.9 and $860.8 \mathrm{eV}$ are attributed to satellite peaks, and the peaks at 870.1 and $855.2 \mathrm{eV}$ can be ascribed to the $\mathrm{Ni}-\mathrm{O}$ bond. The $\mathrm{O}$ in the $\mathrm{Co}-\mathrm{O}$ and $\mathrm{Ni}-\mathrm{O}$ bond may originate from the surface oxidation of NiCo alloy when exposed to air. The characteristic peaks at 871.5 and $853.6 \mathrm{eV}$ can be matched to the metal $\mathrm{Ni}$ in NiCo alloy $[53,54]$.

\subsection{Electromagnetic Performance and Parameter}

According to Eqs. 1 and 2, we can calculate the relationship between the thickness of the corresponding EMWabsorbing material and the reflection loss $(R L)$ in the range of $2-18 \mathrm{GHz}$ frequency. The $R L_{\min }$ value is a key index to evaluate the performance of EMW-absorbing materials. Figures 5 and 6 show 3D and 2D reflection loss diagrams of frequency and thickness correspondence for all samples in the $2-18 \mathrm{GHz}$ range. The $R L_{\min }$ value of $\mathrm{S}-1$ is $-49.01 \mathrm{~dB}$, corresponding to a thickness of $9.8 \mathrm{~mm}$ (Fig. 5a), and the $\mathrm{EAB}_{\text {max }}$ values reaches $3.36 \mathrm{GHz}$ at $5.2 \mathrm{~mm}$ (Fig. 6a), which means the EMW absorption of S-1 performance is not ideal. Figures $5 \mathrm{~b}$ and $6 \mathrm{~b}$ present the EMW-absorbing performance of S-2. However, the $R L_{\min }$ value of $-16.05 \mathrm{~dB}$ and the $\mathrm{EAB}_{\max }$ value of $1.04 \mathrm{GHz}$ indicate that the EMWabsorbing performance of the NiCo-LDHs material also fail to meet the requirements. The corresponding $R L_{\mathrm{min}}$ value of S-3, at a thickness of $4.5 \mathrm{~mm}$, is $-41.46 \mathrm{~dB}$ and the $\mathrm{EAB}_{\max }$ values, at a thickness of $5.1 \mathrm{~mm}$, is $4.0 \mathrm{GHz}$; the reason is that $\mathrm{NiCo}-\mathrm{LDH}$ is transformed into NiCo@C
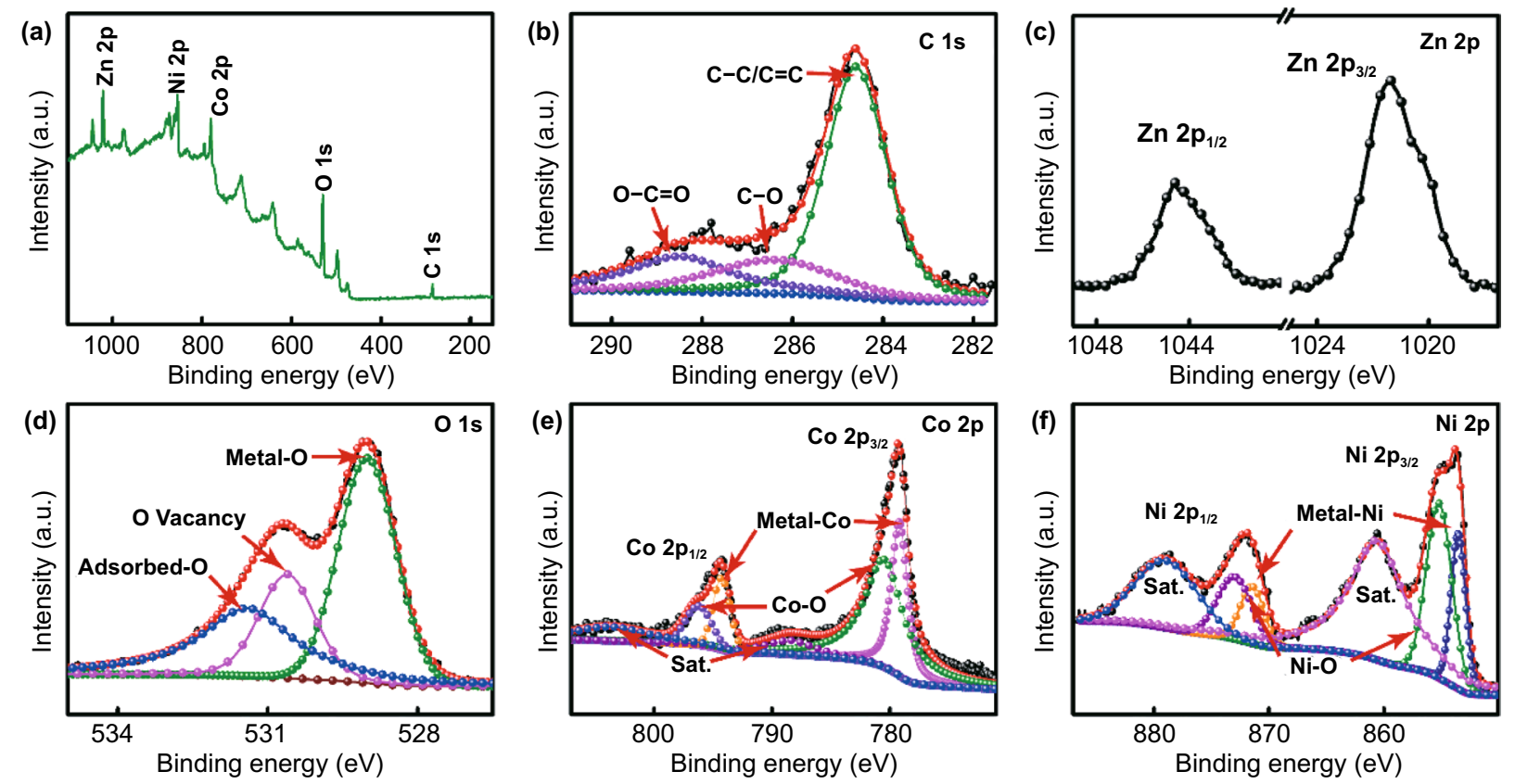

Fig. 4 a Full scan XPS spectra of S-5, b-f high-resolution spectra C 1 s, Zn 2p, O 1 s, Co 2p, and Ni 2p of S-5 

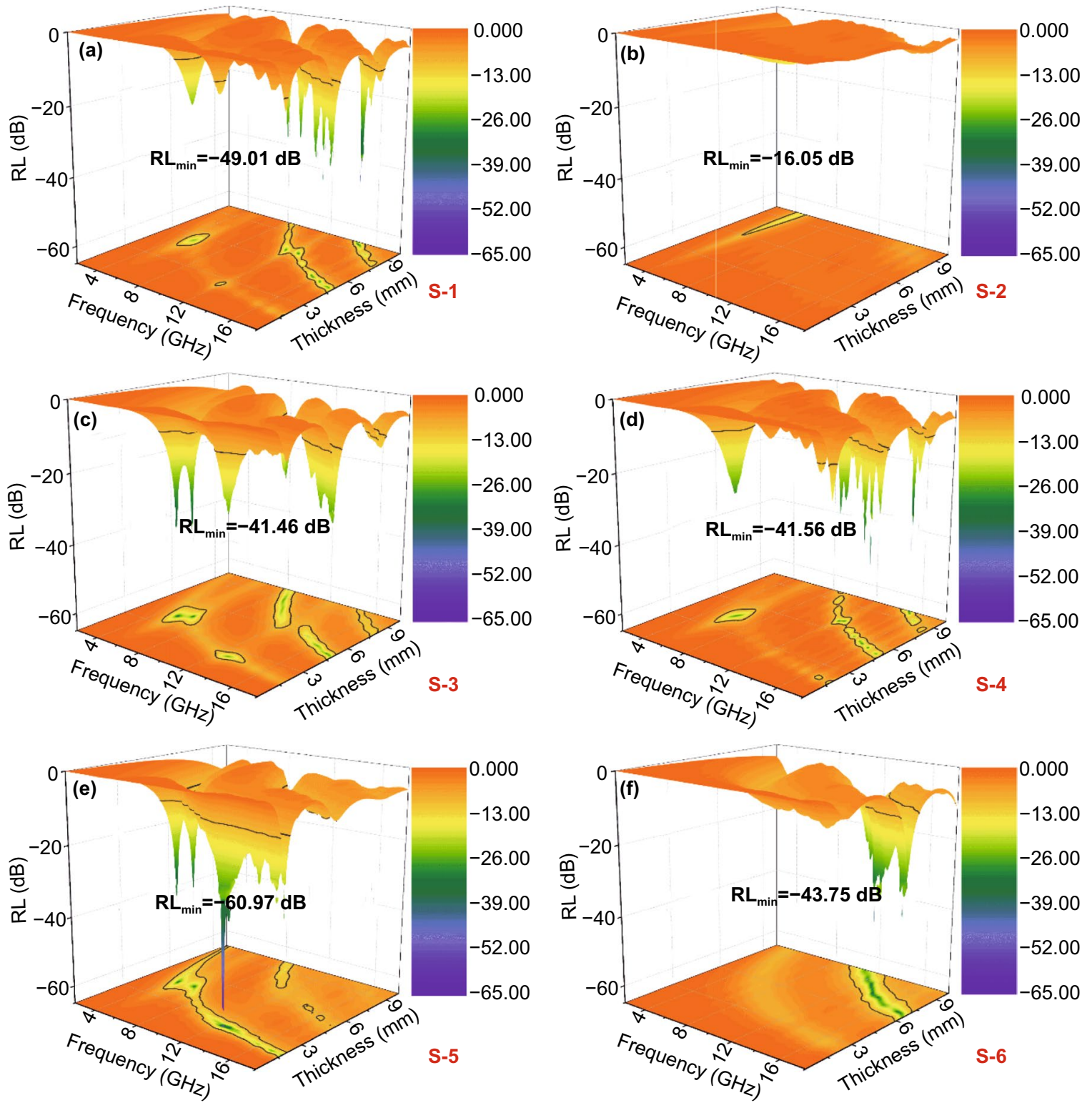

Fig. 5 Reflection loss values in the frequency of 2-18 GHz for a S-1, b S-2, c S-3, d S-4, e S-5 and f S-6

composite by high-temperature annealing, which not only facilitates the construction of conductive network, but also the magnetic loss generated by NiCo alloy can improve the electromagnetic performance. In contrast, after plating the NiCo-LDHs on the surface of rod $\mathrm{ZnO}$, the performance of $\mathrm{S}-4$ has changed not much compared with $\mathrm{S}-1$, the $R L_{\text {min }}$ value of $\mathrm{S}-4$ at $9.9 \mathrm{~mm}$ is $-46.51 \mathrm{~dB}$, and the $\mathrm{EAB}_{\max }$ value is $3.20 \mathrm{GHz}$, which corresponds to the thickness of $5.3 \mathrm{~mm}$.

Figures 5e-f and 6e-f correspond to S-5 and S-6 obtained from calcining S-4 in different atmospheres, respectively. The results showed that the absorption properties of the products obtained by annealing in different inert atmospheres differed significantly. As compared to S-4, the EMW-absorbing performance of S-6 is slightly improved, and the $R L_{\min }$ value drops sharply at a thickness of $7.8 \mathrm{~mm}$ is $-43.75 \mathrm{~dB}$, and the $\mathrm{EAB}_{\text {max }}$ value reaches $4.24 \mathrm{GHz}$ at the thickness of $7.1 \mathrm{~mm}$. However, the EMW absorption performance of S-5 is significantly increased. The $R L_{\min }$ value has reached $-60.97 \mathrm{~dB}$, but the matching thickness is $2.3 \mathrm{~mm}$. The $\mathrm{EAB}_{\max }$ value has reached $6.08 \mathrm{GHz}$, and the matching thickness is only $2.0 \mathrm{~mm}$. 

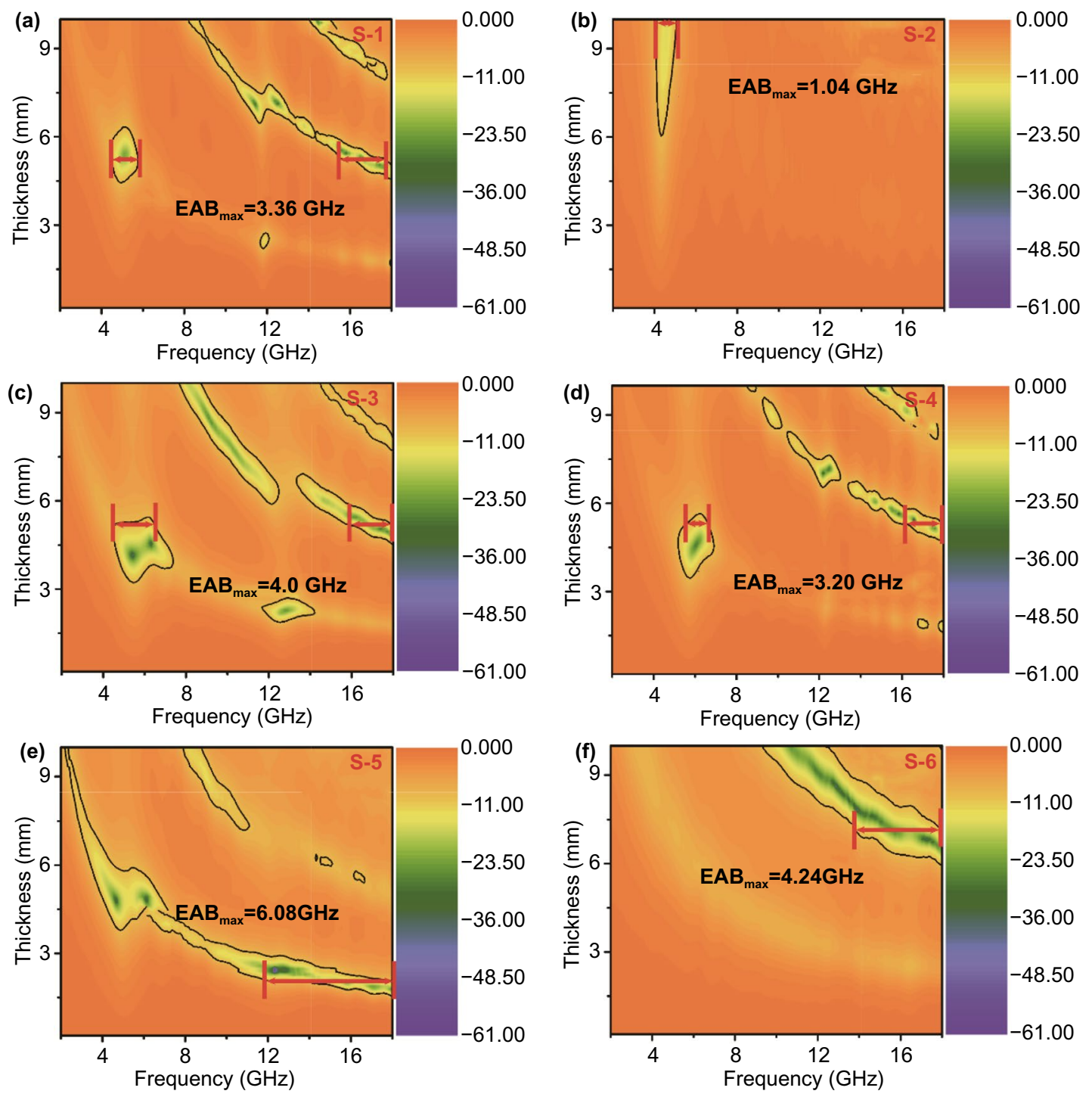

Fig. 6 2D reflection loss diagram of a S-1, b S-2, c S-3, d S-4, e S-5, f S-6

This is because the formation of $\mathrm{NiCo} @ \mathrm{C}$ composites makes significant changes in the structure of S-5, the increase in layer spacing makes the reflection and scattering of incident EMW more frequently, and the appearance of $\mathrm{NiCo@C} \mathrm{composites}$ particles also promotes the polarization loss of incident EMW.

\subsection{Microwave Absorption and Mechanism}

The EM wave absorption characteristics of samples is usually analyzed according to the complex permittivity $\left(\varepsilon_{\mathrm{r}}=\varepsilon^{\prime}-j \varepsilon^{\prime \prime}\right)$ and the complex permeability $\left(\mu_{r}=\mu^{\prime}-j \mu^{\prime \prime}\right)$ [55]. As shown in Fig. 7a, the real part of complex permittivity for S-5 is larger than that of other samples, and the $\varepsilon^{\prime}$ value decreases gradually from 8.7 to 7.0 with the increase in frequency. It has typical dielectric response characteristics [56]. The $\varepsilon^{\prime}$ values of S-1 and S-4 gradually increase with the increase in frequency, which is associated with the presence of $\mathrm{ZnO}$. The $\varepsilon^{\prime}$ values of S-2 and S-3 do not change significantly with frequency, but S-3 shows a significantly improvement than S-2. This is due to weak dielectric loss of NiCo-LDHs and NiCo alloy, which also proves that high-temperature calcination can significantly improve their dielectric properties. In addition, it can also be observed in Fig. 7b-c that the sample has multiple fluctuations at high-frequency bands, which implies that there is a significant polarization loss during the attenuation 
of the incident EMW, which could be due to the interfacial polarization caused by charge accumulation on heterogeneous junction surfaces and the dipole polarization occurring on defects or functional groups of materials. Both polarizations increase the dielectric loss capacity of the absorber [57, 58]. The curves of $\mu^{\prime}$ and $\mu^{\prime \prime}$ versus frequency of all samples (Fig. 7d-e) were observed. A similar trend can be observed for all materials except for S-6, indicating poor magnetic properties. The $\mu^{\prime}$ values of all these materials occur in the range of 4-7 GHz. In general, the large fluctuations in the low-frequency band indicate the presence of natural resonance behavior, while fluctuations in the high-frequency band are attributed to exchange resonance, which contribute magnetic loss for the microwave-absorbing material $[59,60]$.

Debye relaxation is an important way for absorbing materials to have dielectric loss. It can usually be deduced by Eqs. 3 and 4 to obtain Eq. 5, which can express relationship between $\varepsilon^{\prime}$ and $\varepsilon^{\prime \prime}[61,62]$.

$\varepsilon^{\prime}=\varepsilon_{\infty}+\frac{\varepsilon_{S}-\varepsilon_{\infty}}{1+(2 \pi f)^{2} \tau^{2}}$
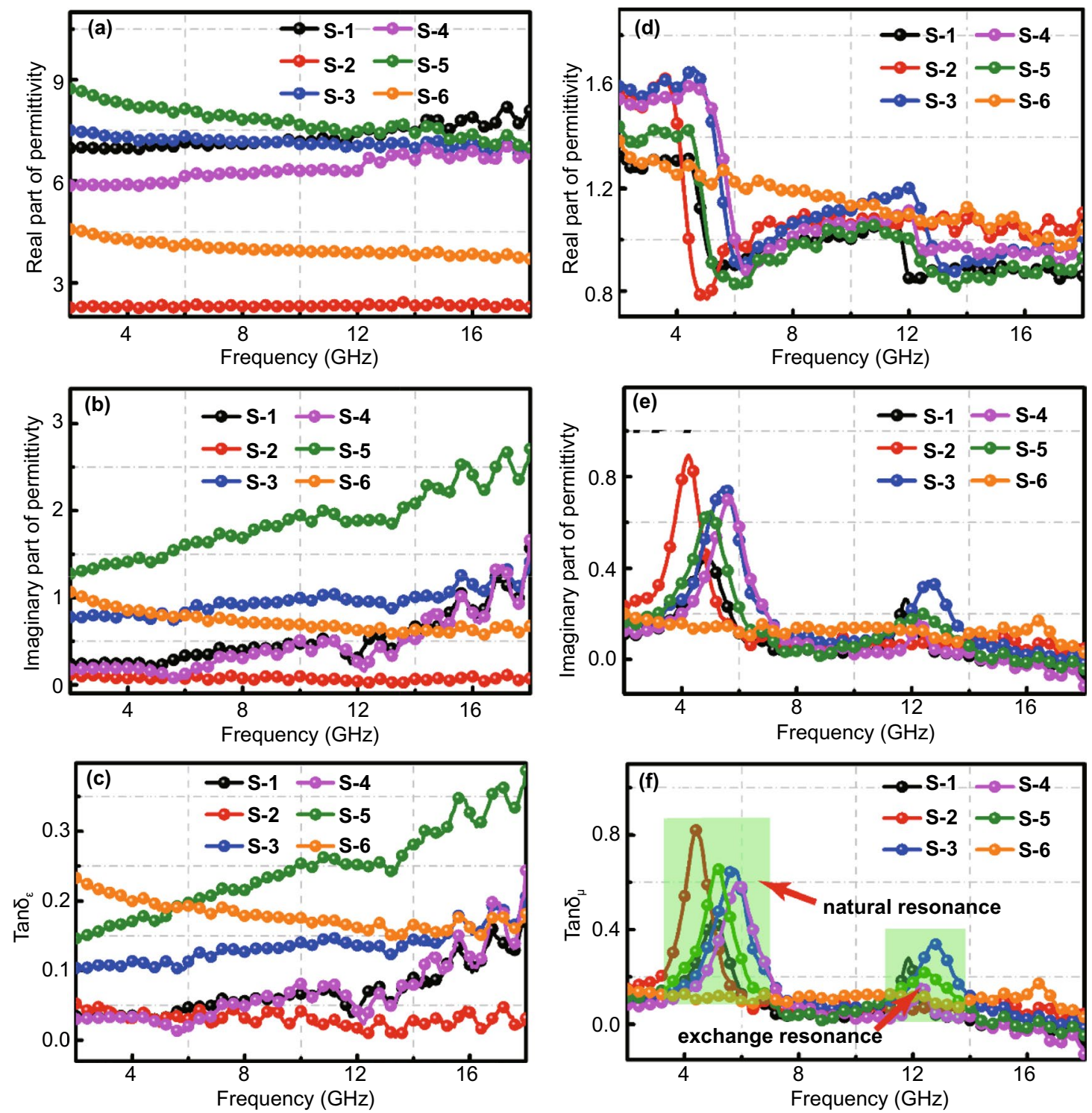

Fig. 7 a Real part and $\mathbf{b}$ imaginary part of complex permittivity of all samples, $\mathbf{d}$ real part and $\mathbf{e}$ imaginary part of the complex permeability of all samples, $\mathbf{c}$ the dielectric loss tangent and $\mathbf{f}$ the magnetic loss tangent of all samples 
$\varepsilon^{\prime \prime}=\frac{2 \pi f \tau\left(\varepsilon_{S}-\varepsilon_{\infty}\right)}{1+(2 \pi f)^{2} \tau^{2}}$

$\left(\varepsilon^{\prime}-\frac{\varepsilon_{S}+\varepsilon_{\infty}}{2}\right)+\left(\varepsilon^{\prime \prime}\right)^{2}=\left(\frac{\varepsilon_{S}-\varepsilon_{\infty}}{2}\right)^{2}$

where $\tau$ stands for polarization relaxation time, $\varepsilon_{s}$ for static permittivity, and $\varepsilon_{\infty}$ points to the high-frequency limited permittivity. As shown in Fig. 8a, more semicircles can be observed in the Cole-Cole diagram of S-5, which implies that more relaxation processes occur in $S-5$, related to the interface between $\mathrm{ZnO} @ \mathrm{C}$ and $\mathrm{ZnO}$. The presence of a variable number of semicircles in other samples can also be seen in Fig. S5, which proves that the Debye relaxation process is prevalent in the prepared material [63]. According to the transmission line theory, the attenuation constant $(\alpha)$ can be calculated by Eq. 6 to reflect the attenuation ability of the absorbing material. Figure $8 \mathrm{~b}$ is the correlation curve between attenuation coefficients and frequencies of all samples. With increasing frequency, all the curves show a significant change, but the attenuation system of S-5 is more prominent than that of other samples, which means that S-5 has a stronger attenuation ability to the incident EMW and shows better absorption performance, which conforms to our previous conclusion [64].

$\alpha=\frac{\sqrt{2} \pi f}{c} \times \sqrt{\left(\mu^{\prime \prime} \varepsilon^{\prime \prime}-\mu^{\prime} \varepsilon^{\prime}\right)+\sqrt{\left(\mu^{\prime} \varepsilon^{\prime \prime}+\mu^{\prime \prime} \varepsilon^{\prime}\right)^{2}+\left(\mu^{\prime \prime} \varepsilon^{\prime \prime}-\mu^{\prime} \varepsilon^{\prime}\right)^{2}}}$

$C_{0}=\mu^{\prime \prime}\left(\mu^{\prime}\right)^{-2} f^{-1}=2 \pi \mu_{0} d^{2} \delta$

where $d$ denotes the thickness of the absorber and $\mu_{0}$ means vacuum permeability. The relationship between $C_{0}$ value and frequency can be used to analyze the mechanism of magnetic loss. When eddy current loss occurs inside the absorbing material, the $C_{0}$ value will not change significantly with the change of frequency [65]. Figure $8 \mathrm{c}$ is the correlation curve between $C_{0}$ and the frequency of all samples. As the frequency increases, the $C_{0}$ value of S-6 does not change much, which indicates that the magnetic loss caused by eddy current loss plays a dominant role, while the other samples have two larger fluctuating peaks at 4-7 and 12-14 GHz, representing natural resonance and exchange resonance respectively. This is consistent with our previous analysis. In the range of 8-11 and $14-18 \mathrm{GHz}$, the variation of $\mathrm{C}_{0}$ value is
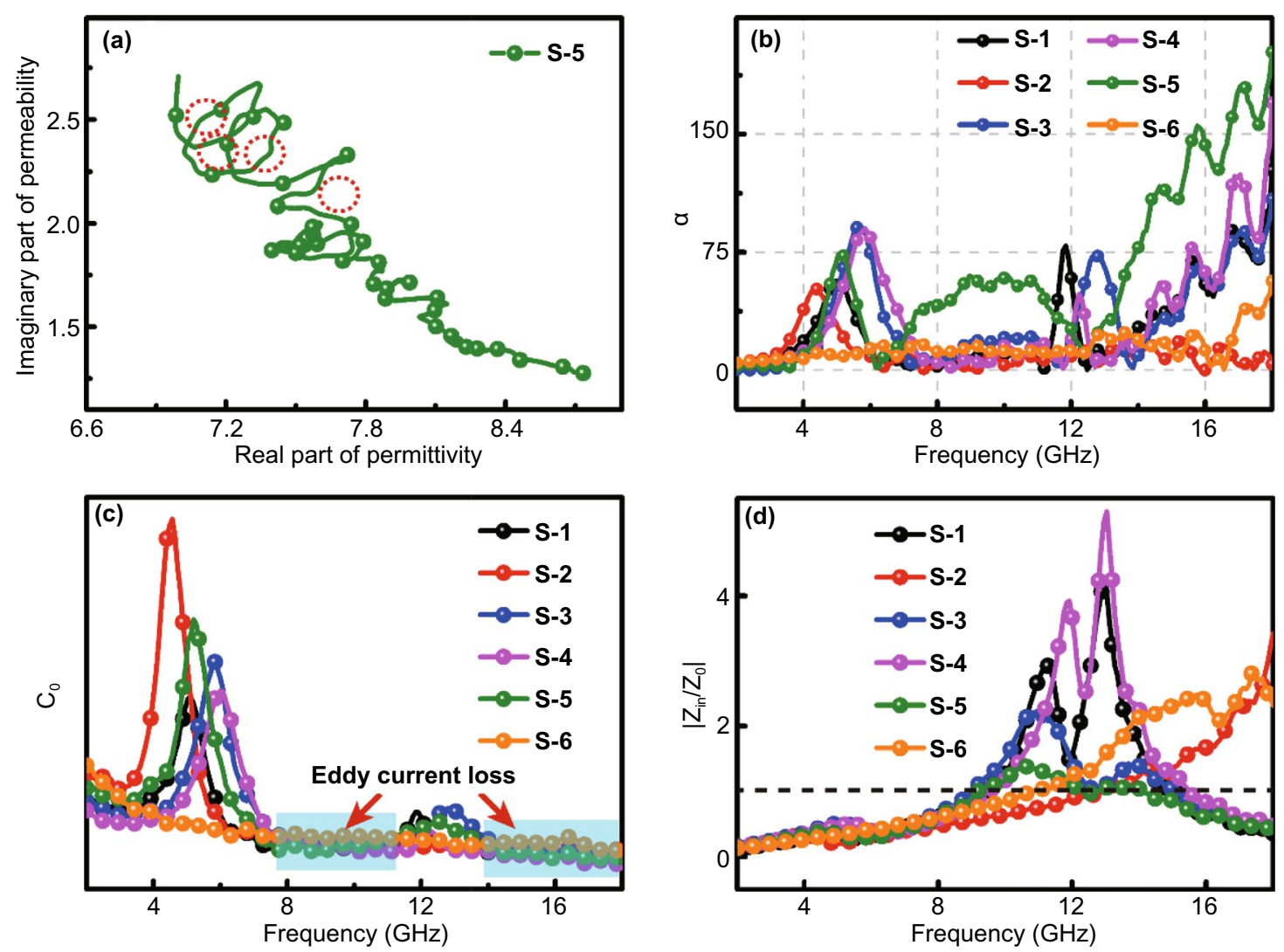

Fig. 8 a Cole-Cole curves of S-5, b attenuation constant of all samples, $\mathbf{c} C_{0}$ of all samples, $\mathbf{d}$ impedance matching at $2.3 \mathrm{~mm}$ of all samples 


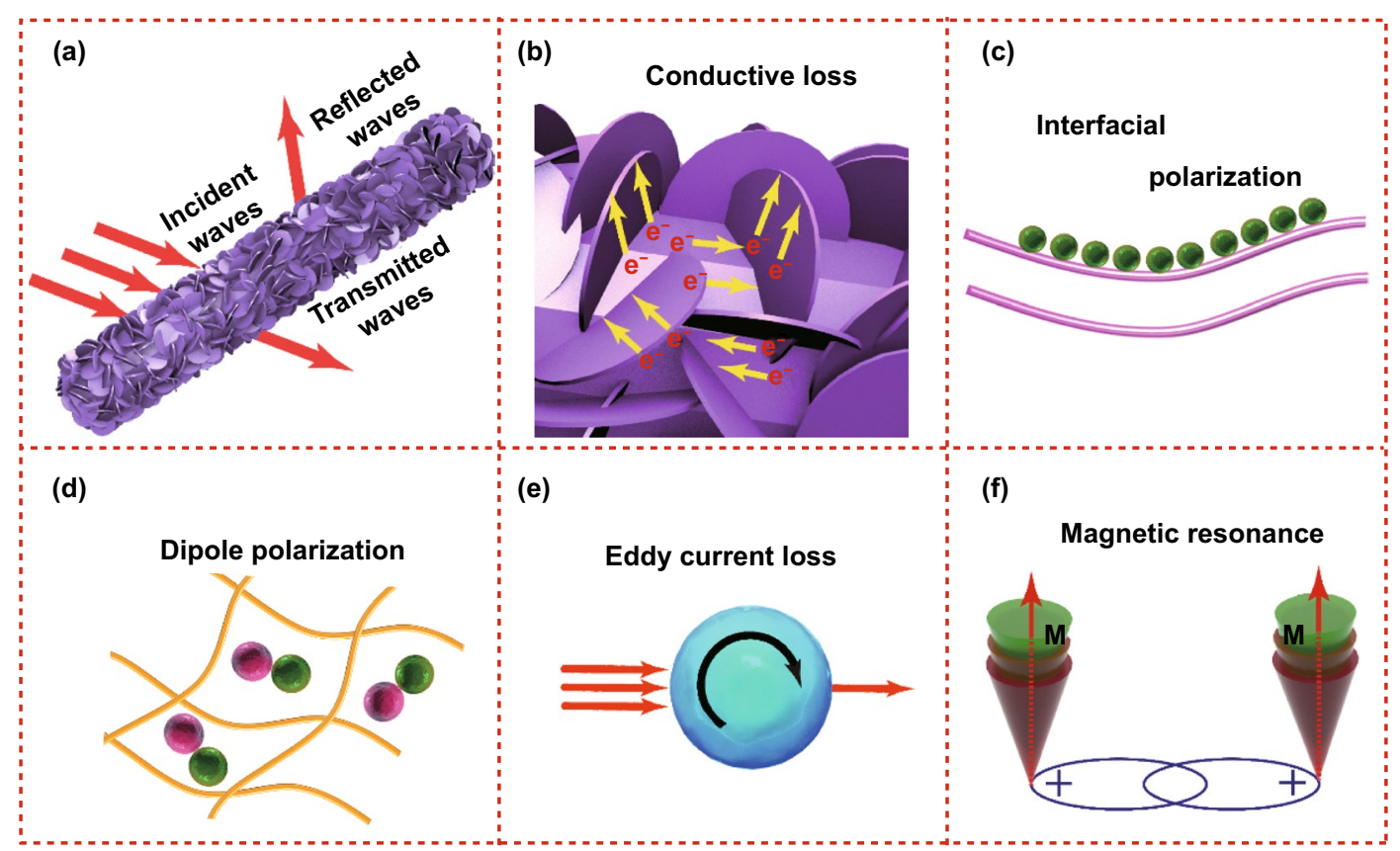

Scheme 2 EM wave absorption mechanism of S-2

Table 1 electromagnetic wave absorption performance of typical composites

\begin{tabular}{|c|c|c|c|c|}
\hline Sample & $R L_{\min }(\mathrm{dB})$ & EAB (GHz) & $d(\mathrm{~mm})$ & References \\
\hline $\mathrm{Ni@C}$ & -59.5 & 4.7 & 2.5 & {$[12]$} \\
\hline Ni@C@ZnO & -55.8 & 4.1 & 2.5 & {$[28]$} \\
\hline $\mathrm{Co} / \mathrm{ZnO} / \mathrm{Ti}_{3} \mathrm{C}_{2} \mathrm{~T}_{\mathrm{x}}$ & -44.22 & 5.28 & 2.4 & {$[30]$} \\
\hline Ni/NiO@C & -51.1 & 5.12 & 2.7 & {$[46]$} \\
\hline $\mathrm{C} / \mathrm{MoS}_{2}$ & -50.1 & 6.0 & 2.2 & {$[58]$} \\
\hline Fe/MnO@C & -45.0 & 5.0 & 2.0 & {$[60]$} \\
\hline CNT-CoFe@C & -40.0 & 5.62 & 2.0 & [71] \\
\hline NiCo@C/ZnO & -60.97 & 6.08 & 2.0 & This work \\
\hline
\end{tabular}

quite small, which indicates that the eddy current loss plays a greater role in the attenuation of electromagnetic waves in this frequency range.

In addition to the attenuation coefficient, impedance matching $\left(\left|Z_{\text {in }} / Z_{0}\right|\right)$ is a critical factor to determine the absorption performance of EMW--absorbing materials. According to Eq. 7, we can get the relationship between $\left|Z_{\text {in }} / Z_{0}\right|$ and frequency of different samples. Generally, the closer the $\left|Z_{\text {in }} / Z_{0}\right|$ value of the EMW-absorbing material is to 1, the EMW materials will absorb EM more easily [66], and the absorbing material will show better EMW absorption performance. From Fig. 8d, it can be found that the curve of S-5 is closer to 1 , which matches its excellent EMW-absorbing performance. The impedance matching values of other materials vary greatly, which means that their impedance matching performance is poor, giving rise to poor EMW absorption performance [67].

Scheme 2 shows a possible EMW absorption mechanism for S-5, which implies that the excellent absorption performance comes as a result of multiple mechanisms. Due to the large specific surface area of the composite material, it is easy to form a conductive network, which is conducive to induced current transmission under the action of the external magnetic field, causing the internal electrons to undergo directional migration and converting electromagnetic energy into thermal energy $[68,69]$. On the one hand, the incident EMW that can enter the material is reflected and scattered, with large amount of EMW attenuating in the process [70]. Secondly, due to the different layered media between $\mathrm{NiCo}$ alloy and rod-like $\mathrm{ZnO}$, electrons will accumulate on the contact interface, leading to interface polarization, which is an important reason for the excellent dielectric parameters of S-5 [71, 72]. Thirdly, the presence of NiCo@C composites and $\mathrm{O}$ vacancies cause dipole polarization in face of an external magnetic field, which promotes the loss of incident EMW. Furthermore, the eddy current loss and resonance loss caused by the presence of NiCo alloy are main sources 
of the magnetic loss [73]. In addition, as shown in Table 1, the composite absorber prepared in this study has better overall absorption performance than other composites.

\section{Conclusion}

In summary, we obtained NiCo-LDHs@ZnO composites through a reasonable combination of NiCo-LDHs with rod-like $\mathrm{ZnO}$ through simple experiments, and the final NiCo@C/ZnO composites were obtained after calcination and had excellent EMW absorption properties. The $R L_{\min }$ value reached $-60.97 \mathrm{~dB}$ at the matching thickness of $2.3 \mathrm{~mm}$, and the $\mathrm{EAB}_{\text {max }}$ value is $6.08 \mathrm{GHz}$ when the matching thickness is $2.0 \mathrm{~mm}$. The interaction of dielectric and magnetic losses is the main reason for the excellent attenuation properties of EMW. This work provides an idea for further expanding the application of LDHs in absorbent materials and provides a method for preparing hetero-structured absorbent materials.

Acknowledgements This work was financially supported by the National Natural Science Foundation of China (No. 51407134), Natural Science Foundation of Shandong Province (No. ZR2019YQ24), Taishan Scholars and Young Experts Program of Shandong Province (No. tsqn202103057), China Postdoctoral Science Foundation (No. 2016M590619) and the Qingchuang Talents Induction Program of Shandong Higher Education Institution (Research and Innovation Team of Structural-Functional Polymer Composites). The authors acknowledge the support from The Thousand Talents Plan, The World-Class University and Discipline, The Taishan Scholar's Advantageous and Distinctive Discipline Program of Shandong Province and The World-Class Discipline Program of Shandong Province.

Open Access This article is licensed under a Creative Commons Attribution 4.0 International License, which permits use, sharing, adaptation, distribution and reproduction in any medium or format, as long as you give appropriate credit to the original author(s) and the source, provide a link to the Creative Commons licence, and indicate if changes were made. The images or other third party material in this article are included in the article's Creative Commons licence, unless indicated otherwise in a credit line to the material. If material is not included in the article's Creative Commons licence and your intended use is not permitted by statutory regulation or exceeds the permitted use, you will need to obtain permission directly from the copyright holder. To view a copy of this licence, visit http://creativecommons.org/licenses/by/4.0/.

Supplementary Information The online version contains supplementary material available at https://doi.org/10.1007/ s40820-021-00704-5.

\section{References}

1. M. Cao, X. Wang, M. Zhang, J. Shu, W. Cao et al., Electromagnetic response and energy conversion for functions and devices in low-dimensional materials. Adv. Funct. Mater. 29(25), 1807398 (2019). https://doi.org/10.1002/adfm.20180 7398

2. F. Zhang, Z. Jia, Z. Wang, C. Zhang, B. Wang et al., Tailoring nanoparticles composites derived from metal-organic framework as electromagnetic wave absorber. Mater. Today Phys. 20, 100475 (2021). https://doi.org/10.1016/j.mtphys. 2021. 100475

3. C. Liang, P. Song, H. Qiu, Y. Zhang, X. Ma et al., Constructing interconnected spherical hollow conductive networks in silver platelets/reduced graphene oxide foam/epoxy nanocomposites for superior electromagnetic interference shielding effectiveness. Nanoscale 11, 22590-22598 (2019). https://doi.org/10. 1039/C9NR06022G

4. H. Lv, Z. Yang, B. Liu, G. Wu, Z. Lou et al., A flexible electromagnetic wave-electricity harvester. Nat. Commun. 12, 834 (2021). https://doi.org/10.1038/s41467-021-21103-9

5. Y. Zhang, K. Ruan, X. Shi, H. Qiu, Y. Pan et al., $\mathrm{Ti}_{3} \mathrm{C}_{2} \mathrm{~T}_{\mathrm{x}} / \mathrm{rGO}$ porous composite films with superior electromagnetic interference shielding performances. Carbon 175, 271-280 (2021). https://doi.org/10.1016/j.carbon.2020.12.084

6. H. Zhang, Z. Jia, A. Feng, Z. Zhou, C. Zhang et al., Enhanced microwave absorption performance of sulfur-doped hollow carbon microspheres with mesoporous shell as a broadband absorber. Compos. Commun. 19, 42-50 (2020). https://doi. org/10.1016/j.coco.2020.02.010

7. G. Ma, L. Xia, H. Yang, X. Wang, T. Zhang et al., Multifunctional lithium aluminosilicate/CNT composite for gas filtration and electromagnetic wave absorption. Chem. Eng. J. 418, 129429 (2021). https://doi.org/10.1016/j.cej.2021.129429

8. C. Zhang, S. Yin, C. Long, B. Dong, D. He et al., Hybrid metamaterial absorber for ultra-low and dual-broadband absorption. Opt. Exp. 29(9), 14078 (2021). https://doi.org/10.1364/ OE.423245

9. Z. Gao, Z. Jia, K. Wang, X. Liu, L. Bi et al., Simultaneous enhancement of recoverable energy density and efficiency of lead-free relaxor-ferroelectric BNT-based ceramics. Chem. Eng. J. 402, 125951 (2020). https://doi.org/10.1016/j.cej.2020. 125951

10. H. Lv, X. Zhou, G. Wu, U. Karaa, X. Wang, Engineering defects in $2 \mathrm{D} \mathrm{g}-\mathrm{C}_{3} \mathrm{~N}_{4}$ for wideband, efficient electromagnetic absorption at elevated temperature. J. Mater. Chem. A (2021). https://doi.org/10.1039/D1TA02785A

11. G. Liu, C. Wu, L. Hu, X. Hu, X. Zhang et al., Anisotropy engineering of metal organic framework derivatives for effective electromagnetic wave absorption. Carbon 181, 48-57 (2021). https://doi.org/10.1016/j.carbon.2021.05.015

12. L. Wang, M. Huang, X. Yu, W. You, J. Zhang et al., MOFderived $\mathrm{Ni}_{1-\mathrm{x}} \mathrm{Co}_{\mathrm{x}} @$ Carbon with tunable nano-microstructure as lightweight and highly efficient electromagnetic wave absorber. Nano-Micro Lett. 12, 150 (2020). https://doi.org/ 10.1007/s40820-020-00488-0 
13. X. Zhou, Z. Jia, X. Zhang, B. Wang, X. Liu et al., Electromagnetic wave absorption performance of $\mathrm{NiCo}_{2} \mathrm{X}_{4}(\mathrm{X}=\mathrm{O}, \mathrm{S}$, $\mathrm{Se}, \mathrm{Te})$ spinel structures. Chem. Eng. J. 420, 129907 (2021). https://doi.org/10.1016/j.cej.2021.129907

14. J. Zhao, J. Zhang, L. Wang, J. Li, T. Feng et al., Superior wave-absorbing performances of silicone rubber composites via introducing covalently bonded $\mathrm{SnO}_{2} @$ MWCNT absorbent with encapsulation structure. Compos. Commun. 22, 100486 (2020). https://doi.org/10.1016/j.coco.2020.100486

15. X. Zhu, Y. Dong, Z. Xiang, L. Cai, F. Pan et al., Morphologycontrollable synthesis of polyurethane-derived highly crosslinked 3D networks for multifunctional and efficient electromagnetic wave absorption. Carbon 182, 254-264 (2021). https://doi.org/10.1016/j.carbon.2021.06.028

16. T. Hou, Z. Jia, B. Wang, H. Li, X. Liu et al., MXene-based accordion 2D hybrid structure with $\mathrm{Co}_{9} \mathrm{~S}_{8} / \mathrm{C} / \mathrm{Ti}_{3} \mathrm{C}_{2} \mathrm{~T}_{\mathrm{x}}$ as efficient electromagnetic wave absorber. Chem. Eng. J. 414, 128875 (2021). https://doi.org/10.1016/j.cej.2021.128875

17. P. Liu, S. Gao, Y. Wang, Y. Huang, Y. Wang et al., Core-shell CoNi@graphitic carbon decorated on B, N-Co doped hollow carbon polyhedrons toward lightweight and high-efficiency microwave attenuation. ACS Appl. Mater. Interfaces 11(28), 25624-25635 (2019). https://doi.org/10.1021/acsami.9b085 25

18. S. Hu, Y. Zhou, M. He, Q. Liao, H. Yang et al., Hollow Ni-Co layered double hydroxides-derived NiCo-alloy@g- $\mathrm{C}_{3} \mathrm{~N}_{4}$ microtubule with high performance microwave absorption. Mater. Lett. 231, 171-174 (2018). https://doi.org/10.1016/j. matlet.2018.08.048

19. L. Wang, B. Wen, X. Bai, C. Liu, H. Yang, NiCo alloy/carbon nanorods decorated with carbon nanotubes for microwave absorption. ACS Appl. Nano Mater. 2(12), 7827-7838 (2019). https://doi.org/10.1021/acsanm.9b01842

20. H. Sun, C. Tian, Y. Li, J. Wu, Q. Wang et al., Coupling NiCo alloy and $\mathrm{CeO}_{2}$ to enhance electrocatalytic hydrogen evolution in alkaline solution. Adv. Sustain. Syst. 4(11), 2000122 (2020). https://doi.org/10.1002/adsu.202000122

21. X. Gao, Z. Jia, B. Wang, X. Wu, T. Sun et al., Synthesis of NiCo-LDH/MXene hybrids with abundant heterojunction surfaces as a lightweight electromagnetic wave absorber. Chem. Eng. J. 419, 130019 (2021). https://doi.org/10.1016/j.cej.2021. 130019

22. Z. Zhang, Z. Cai, Z. Wang, Y. Peng, L. Xia et al., A review on metal-organic framework derived porous carbon-based novel microwave absorption materials. Nano-Micro Lett. 13, 56 (2021). https://doi.org/10.1007/s40820-020-00582-3

23. W. Cheng, Y. Wang, S. Ge, X. Ding, Z. Cui et al., One-step microwave hydrothermal preparation of $\mathrm{Cd} / \mathrm{Zr}$-bimetallic metal-organic frameworks for enhanced photochemical properties. Adv. Compos. Hybrid. Mater. 4, 150-161 (2021). https://doi.org/10.1007/s42114-020-00199-5

24. T. Hou, Z. Jia, B. Wang, H. Li, X. Liu et al., Metal-organic framework-derived $\mathrm{NiSe}_{2}-\mathrm{CoSe}_{2} @ \mathrm{C} / \mathrm{Ti}_{3} \mathrm{C}_{2} \mathrm{~T}_{\mathrm{x}}$ composites as electromagnetic wave absorbers. Chem. Eng. J. 422, 130079 (2021). https://doi.org/10.1016/j.cej.2021.130079
25. P. Liu, S. Gao, Y. Wang, F. Zhou, Y. Huang et al., Metalorganic polymer coordination materials derived $\mathrm{Co} / \mathrm{N}$-doped porous carbon composites for frequency-selective microwave absorption. Compos. Part B 202, 108406 (2020). https://doi. org/10.1016/j.compositesb.2020.108406

26. B. Zhong, L. Zhang, J. Yu, K. Fan, Ultrafine iron-cobalt nanoparticles embedded in nitrogen-doped porous carbon matrix for oxygen reduction reaction and zinc-air batteries. J. Colloid Interface Sci. 546, 113-121 (2019). https://doi.org/10.1016/j. jcis.2019.03.038

27. Y. Wang, S. Yang, H. Wang, G. Wang, X. Sun et al., Hollow porous $\mathrm{CoNi} / \mathrm{C}$ composite nanomaterials derived from MOFs for efficient and lightweight electromagnetic wave absorber. Carbon 167, 485-494 (2020). https://doi.org/10.1016/j.carbon. 2020.06.014

28. L. Wang, X. Yu, X. Li, J. Zhang, M. Wang et al., MOFderived yolk-shell Ni@C@ZnO schottky contact structure for enhanced microwave absorption. Chem. Eng. J. 383, 123099 (2020). https://doi.org/10.1016/j.cej.2019.123099

29. Q. Liao, M. He, Y. Zhou, S. Nie, Y. Wang et al., Highly cuboid-shape heterobimetallic metal-organic frameworks derived from porous $\mathrm{Co} / \mathrm{ZnO} / \mathrm{C}$ microrods with improved electromagnetic wave absorption capabilities. ACS Appl. Mater. Interface 10(34), 29136-29144 (2018). https://doi.org/10. 1021/acsami.8b09093

30. M. Kong, Z. Jia, B. Wang, J. Dou, X. Liu et al., Construction of metal-organic framework derived $\mathrm{Co} / \mathrm{ZnO} / \mathrm{Ti}_{3} \mathrm{C}_{2} \mathrm{~T}_{\mathrm{x}}$ composites for excellent microwave absorption. Sustain. Mater. Technol. 26, e00219 (2020). https://doi.org/10.1016/j.susmat. 2020.e00219

31. L. Wang, X. Shi, J. Zhang, Y. Zhang, J. Gu, Lightweight and robust $\mathrm{rGO} /$ sugarcane derived hybrid carbon foams with outstanding EMI shielding performance. J. Mater. Sci. Technol. 52, 119-126 (2020). https://doi.org/10.1016/j.jmst.2020.03. 029

32. Z. Jia, Z. Gao, K. Kou, A. Feng, C. Zhang et al., Facile synthesis of hierarchical a-site cation deficiency perovskite $\mathrm{La}_{\mathrm{x}} \mathrm{FeO}_{3-\mathrm{y}} / \mathrm{RGO}$ for high efficiency microwave absorption. Compos. Commun. 20, 100344 (2020). https://doi.org/10. 1016/j.coco.2020.04.010

33. L. Cao, J. Wang, D. Zhong, T. Lu, Template-directed synthesis of sulphur doped $\mathrm{NiCoFe}$ layered double hydroxide porous nanosheets with enhanced electrocatalytic activity for the oxygen evolution reaction. J. Mater. Chem. A 6(7), 3224-3232 (2018). https://doi.org/10.1039/C7TA09734D

34. F. Zhu, W. Liu, Y. Liu, W. Shi, Construction of porous interface on CNTs@NiCo-LDH core-shell nanotube arrays for supercapacitor applications. Chem. Eng. J. 383, 123150 (2020). https://doi.org/10.1016/j.cej.2019.123150

35. R. Zhang, R. Zhu, Y. Li, Z. Hui, Y. Song et al., CoP and $\mathrm{Ni}_{2} \mathrm{P}$ implanted in hollow porous $\mathrm{N}$-doped carbon polyhedron for $\mathrm{pH}$ universal hydrogen evolution reaction and alkaline overall water splitting. Nanoscale 12(46), 23851-23858 (2020). https://doi.org/10.1039/D0NR07126A

36. X. Kong, B. Xia, Y. Xiao, H. Chen, H. Li et al., Regulation of Cobalt-Nickel LDHs' structure and components for optimizing 
the performance of electrochemical sensor. ACS Appl. Nano Mater. 2(10), 6387-6396 (2019). https://doi.org/10.1021/ acsanm.9b01370

37. Y. Dou, J. Zhou, F. Yang, M. Zhao, Z. Nie et al., Hierarchically structured Layered-double-hydroxide@Zeolitic-imidazolateframework derivatives for high-performance electrochemical energy storage. J. Mater. Chem. A 4(32), 12526-12134 (2016). https://doi.org/10.1039/C6TA04765C

38. Y. Xie, C. Feng, Y. Guo, S. Li, C. Guo et al., MOFs derived carbon nanotubes coated $\mathrm{CoNi}$ alloy nanocomposites with $\mathrm{N}$-doped rich-defect and abundant cavity structure as efficient trifunctional electrocatalyst. Appl. Surf. Sci. 53, 147786 (2021). https://doi.org/10.1016/j.apsusc.2020.147786

39. J. Kang, H. Zhang, X. Duan, H. Sun, X. Tan et al., Magnetic $\mathrm{Ni}-\mathrm{Co}$ alloy encapsulated $\mathrm{N}$-doped carbon nanotubes for catalytic membrane degradation of emerging contaminants. Chem. Eng. J. 362, 251-261 (2019). https://doi.org/10.1016/j.cej. 2019.01.035

40. J. Wang, B. Wang, Z. Wang, L. Chen, C. Gao et al., Synthesis of $3 \mathrm{D}$ flower-like $\mathrm{ZnO} / \mathrm{ZnCo}_{2} \mathrm{O}_{4}$ composites with the heterogeneous interface for excellent electromagnetic wave absorption properties. J. Colloid Interface Sci. 586, 479-490 (2021). https://doi.org/10.1016/j.jcis.2020.10.111

41. Y. Dong, X. Zhu, F. Pan, B. Deng, Z. Liu et al., Mace-like carbon fiber/ZnO nanorod composite derived from typha orientalis for lightweight and high-efficient electromagnetic wave absorber. Adv. Compos. Hybrid. Mater. (2021). https://doi.org/ 10.1007/s42114-021-00277-2

42. M. Qin, L. Zhang, X. Zhao, H. Wu, Defect induced polarization loss in multi-shelled spinel hollow spheres for electromagnetic wave absorption application. Adv. Sci. 8, 2004640 (2021). https://doi.org/10.1002/advs.202004640

43. X. Zhou, Z. Jia, A. Feng, K. Wang, X. Liu et al., Dependency of tunable electromagnetic wave absorption performance on morphology-controlled 3D porous carbon fabricated by biomass. Compos. Commun. 21, 100404 (2020). https://doi.org/ 10.1016/j.coco.2020.100404

44. H. Li, Q. Jin, J. Zhao, B. Wang, X. Guo, Rational synthesis of ZIF-67@Co-Ni LDHs heterostructure and derived heterogeneous carbon-based framework as high-efficient multifunctional sulfur host. Dalton. Trans. 49(36), 12686-12694 (2020). https://doi.org/10.1039/D0DT02442B

45. Y. Liu, Y. Wang, C. Shi, Y. Chen, D. Li et al., Co-ZIF derived porous NiCo-LDH nanosheets/N doped carbon foam for high performance supercapacitor. Carbon 165, 129-138 (2020). https://doi.org/10.1016/j.carbon.2020.04.084

46. X. Zhou, Z. Jia, X. Zhang, B. Wang, W. Wu et al., Controllable synthesis of $\mathrm{Ni} / \mathrm{NiO} @$ porous carbon hybrid composites towards remarkable electromagnetic wave absorption and wide absorption bandwidth. J. Mater. Sci. Technol. 87, 120-132 (2021). https://doi.org/10.1016/j.jmst.2021.01.073

47. X. Liang, Z. Man, B. Quan, J. Zheng, W. Gu et al., Environment-stable $\mathrm{Co}_{\mathrm{x}} \mathrm{Ni}_{\mathrm{y}}$ encapsulation in stacked porous carbon nanosheets for enhanced microwave absorption. Nano-Micro Lett. 12, 102 (2020). https://doi.org/10.1007/ s40820-020-00432-2
48. H. Yan, X. Dai, K. Ruan, S. Zhang, X. Shi et al., Flexible thermally conductive and electrically insulating silicone rubber composite films with BNNS@ $\mathrm{Al}_{2} \mathrm{O}_{3}$ fillers. Adv. Compos. Hybrid. Mater. 4, 36-50 (2021). https://doi.org/10.1007/ s42114-021-00208-1

49. T. Hou, Z. Jia, A. Feng, Z. Zhou, X. Liu et al., Hierarchical composite of biomass derived magnetic carbon framework and phytic acid doped polyanilne with prominent electromagnetic wave absorption capacity. J. Mater. Sci. Technol. 68, 61-69 (2021). https://doi.org/10.1016/j.jmst.2020.06.046

50. J. Zhao, J. Zhang, L. Wang, S. Lyu, W. Ye et al., Fabrication and investigation on ternary heterogeneous MWCNT@ $\mathrm{TiO}_{2}-\mathrm{C}$ fillers and their silicone rubber wave-absorbing composites. Compos. Part A 129, 105714 (2020). https://doi.org/10.1016/j. compositesa.2019.105714

51. Q. Chang, H. Liang, B. Shi, H. Wu, Sodium oxalate-induced hydrothermal synthesis of wood-texture-column-like $\mathrm{NiCo}_{2} \mathrm{O}_{4}$ with broad bandwidth electromagnetic wave absorption performance. J. Colloid Interface Sci. 600, 49-57 (2021). https:// doi.org/10.1016/j.jcis.2021.05.019

52. X. Zhang, J. Zhu, P. Yin, A. Guo, A. Huang et al., Tunable high-performance microwave absorption of $\mathrm{Co}_{1-\mathrm{x}} \mathrm{S}$ hollow spheres constructed by nanosheets within ultralow filler loading. Adv. Funct. Mater. 28(49), 1800761 (2018). https://doi. org/10.1002/adfm.201800761

53. S. Gao, G. Zhang, Y. Wang, X. Han, Y. Huang et al., MOFs derived magnetic porous carbon microspheres constructed by core-shell Ni@C with high-performance microwave absorption. J. Mater. Sci. Technol. 88, 56-65 (2021). https://doi.org/ 10.1016/j.jmst.2021.02.011

54. B. Zhao, Y. Li, X. Guo, R. Zhang, J. Zhang et al., Enhanced electromagnetic wave absorbing nickel (oxide)-carbon nanocomposites. Ceram. Int. 45(18), 24474-24486 (2019). https:// doi.org/10.1016/j.ceramint.2019.08.173

55. M. Green, Z. Liu, R. Smedley, H. Nawaz, X. Li et al., Graphitic carbon nitride nanosheets for microwave absorption. Mater. Today Phys. 5, 78-86 (2018). https://doi.org/10.1016/j. mtphys.2018.06.005

56. S. Dong, W. Zhang, X. Zhang, P. Hu, J. Han, Designable synthesis of core-shell $\mathrm{SiC}_{\mathrm{w}} @ \mathrm{C}$ heterostructures with thickness-dependent electromagnetic wave absorption between the whole X-band and Ku-band. Chem. Eng. J. 354, 767-776 (2018). https://doi.org/10.1016/j.cej.2018.08.062

57. H. Zhang, Z. Jia, B. Wang, X. Wu, T. Sun et al., Construction of remarkable electromagnetic wave absorber from heterogeneous structure of $\mathrm{Co}-\mathrm{CoFe}_{2} \mathrm{O}_{4} @$ mesoporous hollow carbon spheres. Chem. Eng. J. 421, 129960 (2021). https://doi.org/10. 1016/j.cej.2021.129960

58. F. Pan, Z. Liu, B. Deng, Y. Dong, X. Zhu et al., Lotus leafderived gradient hierarchical porous $\mathrm{C} / \mathrm{MoS}_{2}$ morphology genetic composites with wideband and tunable electromagnetic absorption performance. Nano-Micro Lett. 13, 43 (2021). https://doi.org/10.1007/s40820-020-00568-1

59. M. Green, J. Xu, H. Liu, J. Zhao, K. Li et al., Terahertz absorption of hydrogenated $\mathrm{TiO}_{2}$ nanoparticles. Mater. Today Phys. 4, 64-69 (2018). https://doi.org/10.1016/j.mtphys.2018.04.001 
60. G. He, Y. Duan, H. Pang, Microwave absorption of crystalline $\mathrm{Fe} / \mathrm{MnO} @ \mathrm{C}$ nanocapsules embedded in amorphous carbon. Nano-Micro Lett. 12, 57 (2020). https://doi.org/10.1007/ s40820-020-0388-4

61. Z. Lou, C. Yuan, Y. Zhang, Y. Li, J. Cai et al., Synthesis of porous carbon matrix with inlaid $\mathrm{Fe}_{3} \mathrm{C} / \mathrm{Fe}_{3} \mathrm{O}_{4}$ micro-particles as an effective electromagnetic wave absorber from natural wood shavings. J. Alloy Compd. 775, 800-809 (2019). https:// doi.org/10.1016/j.jallcom.2018.10.213

62. Z. Gao, Z. Zhao, D. Lan, K. Kou, J. Zhang et al., Accessory ligand strategies for hexacyanometallate networks deriving perovskite polycrystalline electromagnetic absorbents. J. Mater. Sci. Technol. 82, 69-79 (2021). https://doi.org/10. 1016/j.jmst.2020.11.071

63. H. Lv, Y. Li, Z. Jia, L. Wang, X. Guo et al., Exceptionally porous three-dimensional architectural nanostructure derived from CNTs/graphene aerogel towards the ultra-wideband EM absorption. Compos. Part B 196, 108122 (2020). https://doi. org/10.1016/j.compositesb.2020.108122

64. J. Xu, L. Xia, J. Luo, S. Lu, X. Huang et al., High-performance electromagnetic wave absorbing $\mathrm{CNT} / \mathrm{SiC}_{\mathrm{f}}$ composites: synthesis, tuning, and mechanism. ACS Appl. Mater. Interfaces 12, 20775-20784 (2020). https://doi.org/10.1021/acsami. 9b19281

65. X. Zhang, X. Zhang, D. Wang, H. Yuan, S. Zhang et al., Three-dimensional graphene-supported nitrogen-doped carbon nanotube architecture for attenuation of electromagnetic energy. J. Mater. Chem. C 7(38), 11868-11878 (2019). https:// doi.org/10.1039/C9TC04191E

66. G. Liu, J. Tu, C. Wu, Y. Fu, C. Chu et al., High-yield twodimensional metal-organic framework derivatives for wideband electromagnetic wave absorption. ACS Appl. Mater. Interfaces 13, 20459-20466 (2021). https://doi.org/10.1021/ acsami.1c00281
67. D. Zhang, H. Zhang, J. Cheng, H. Raza, T. Liu et al., Customizing coaxial stacking $\mathrm{VS}_{2}$ nanosheets for dual-band microwave absorption with superior performance in the $\mathrm{C}$ - and $\mathrm{Ku}$ bands. J. Mater. Chem. C 8, 5923-5933 (2020). https://doi. org/10.1039/D0TC00763C

68. D. Lan, Z. Gao, Z. Zhao, K. Kou, H. Wu, Application progress of conductive conjugated polymers in electromagnetic wave absorbing composites. Comp. Commun. 26, 100767 (2021). https://doi.org/10.1016/j.coco.2021.100767

69. H. Lv, C. Wu, F. Qin, H. Peng, M. Yan, Extra-wide bandwidth via complementary exchange resonance and dielectric polarization of sandwiched FeNi@ $\mathrm{SnO}_{2}$ nanosheets for electromagnetic wave absorption. J. Mater. Sci. Technol. 90, 1-8 (2021). https://doi.org/10.1016/j.jmst.2020.12.083

70. M. Xu, J. Liu, H. Zhang, Y. Zhang, X. Wu et al., Electrically conductive $\mathrm{Ti}_{3} \mathrm{C}_{2} \mathrm{~T}_{\mathrm{x}}$ MXene/polypropylene nanocomposites with an ultralow percolation threshold for efficient electromagnetic interference shielding. Ind. Eng. Chem. Res. 60, 4342-4350 (2021). https://doi.org/10.1021/acs.iecr.1c00320

71. Z. Wang, L. Yang, Y. Zhou, C. Xu, M. Yan et al., NiFe LDH/ MXene derivatives interconnected with carbon fabric for flexible electromagnetic wave absorption. ACS Appl. Mater. Interfaces 13, 16713-16721 (2021). https://doi.org/10.1021/ acsami.1c05007

72. M. Qin, L. Zhang, X. Zhao, H. Wu, Lightweight Ni foambased ultra-broadband electromagnetic wave absorber. Adv. Funct. Mater. (2021). https://doi.org/10.1002/adfm.202103436

73. L. Liang, Q. Li, X. Yan, Y. Feng, Y. Wang et al., Multifunctional magnetic $\mathrm{Ti}_{3} \mathrm{C}_{2} \mathrm{~T}_{\mathrm{x}}$ MXene/graphene aerogel with superior electromagnetic wave absorption performance. ACS Nano 15, 6622-6632 (2021). https://doi.org/10.1021/acsnano.0c099 82 